U. Einhorn-Stoll, H. Kastner, B. Senge

\title{
Comparison of Molecular Parameters; Material Properties and Gelling behaviour of Commercial Citrus Pectins
}

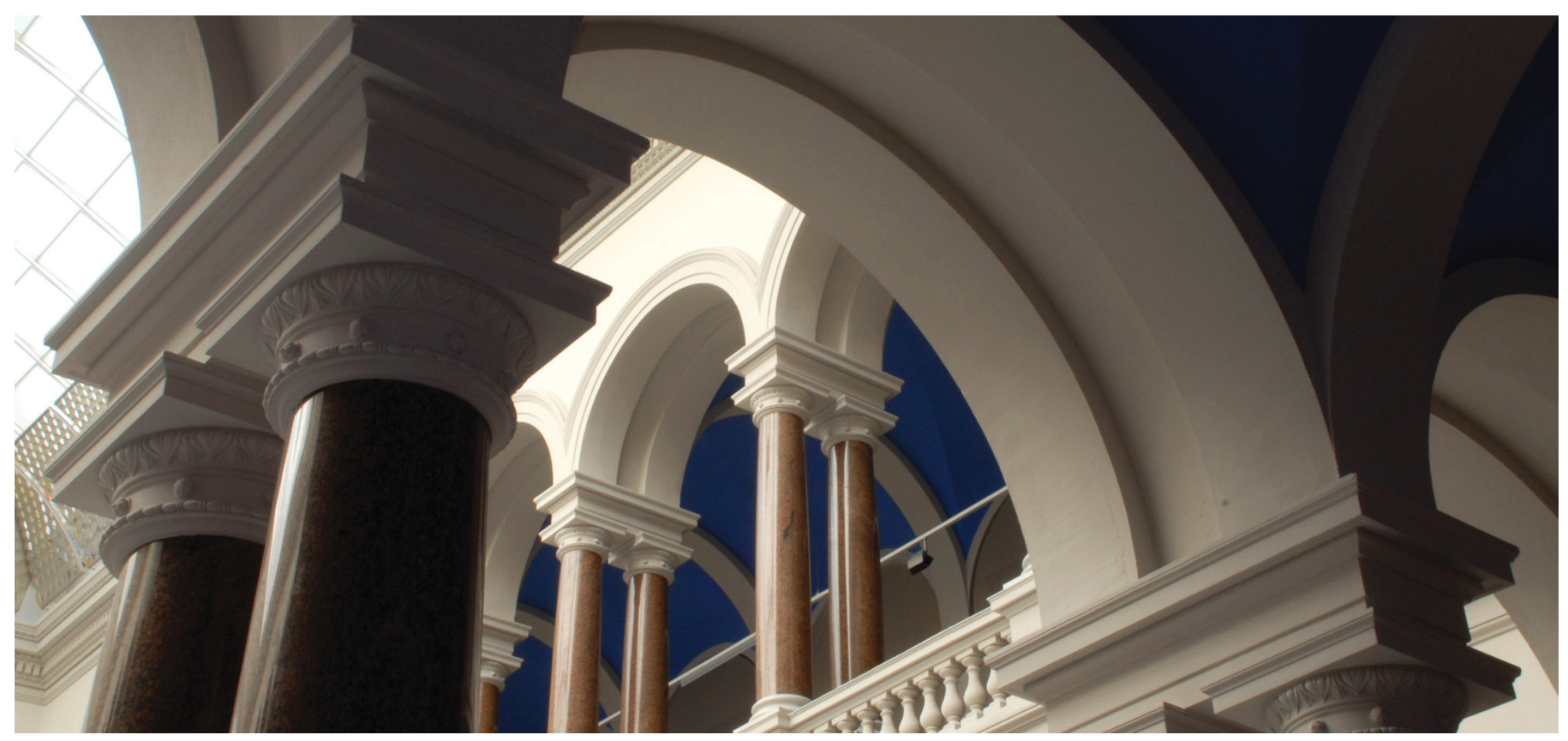

Einhorn-Stoll, U., Kastner, H., \& Senge, B. (2012). Comparison of Molecular Parameters; Material Properties and Gelling behaviour of Commercial Citrus Pectins. In Gums and Stabilisers for the Food Industry 16 (pp. 199-206). https://doi.org/10.1039/9781849734554-00199 
COMPARISON OF MOLECULAR PARAMETERS; MATERIAL PROPERTIES AND GELLING BEHAVIOUR OF COMMERCIAL CITRUS PECTINS

\author{
U. Einhorn-Stoll, H. Kastner, B. Senge \\ Department of Food Technology and Food Chemistry, Technische Universitaet Berlin, \\ Koenigin-Luise-Strasse 22, D-14195 Berlin, Germany
}

\title{
1 INTRODUCTION
}

Pectins are important gelling and thickening agents for the food industry. They are extracted mainly from citrus fruits and apples but also from different other plant materials such as sunflower, sugar beet or other sources. The industrial production has a long tradition and the main steps like extraction or precipitation are well-known. ${ }^{1}$ Nevertheless, the parameters and details of the treatments, such as temperatures, $\mathrm{pH}$ or drying procedure, can vary considerably between different pectin producing companies and even within one company. Moreover, the pectin sources are biological materials with seasonal and local variations, and it is necessary but not always completely possible for the pectin producers to adapt the technology to the raw material.

Pectin molecules are long mainly galacturonic acid backbones with side chains of neutral sugars. The galacturonic acid molecules are partly methoxylated and the pectins are divided into high-methoxylated (HMP) or low-methoxylated (LMP) with degree of methoxylation (DM) above or below $50 \%$, respectively. ${ }^{1,2,3}$ LMP are mostly made from HMP by chemical demethoxylation procedures with acidic or alkaline conditions; ${ }^{1}$ the resulting pectins are used for different food products.

In previous experiments, pectin modifications such as demethoxylation and amidation were made from HM-pectin of one company in laboratory scale. It was found that the material properties and thermal degradation behaviour of the resulting LMP varied considerably in dependence on their molecular parameters and preparation conditions. ${ }^{4,5}$ Laboratory preparation and industrial production differ, however, not only with respect to the raw material properties and amount of processed material but also in the applied equipment and resulting technological conditions. The question is, whether results of model pectins can be transferred to industrially produced materials from different companies. Therefore, commercial citrus pectins from three different companies were examined in detail and compared with respect to their molecular and material properties and especially their gelling behaviour. These parameters are relevant for practical pectin application and their interactions and inter-dependencies can give valuable information for pectin producing as well as using companies. 


\section{MATERIALS AND METHODS}

\subsection{Materials}

All samples were commercial pectins, kindly provided from three pectin companies. For data protection reasons they are named with 1, 2 and 3, and the pectins are labelled with 1A, $1 \mathrm{~B}$, etc. The detailed examined parameters are given in Table 1.

\subsection{Methods}

The molecular parameters galacturonan content GC, degree of methoxylation DM, intrinsic viscosity IV and the material properties colour and dissolution time as well as thermal degradation properties were determined as described previously. ${ }^{5,6}$ The gelling behaviour was tested by oscillation measurements with temperature sweep as described in a parallel paper. $^{7}$ The particle size of the dry powder was determined using a Horiba particle sizer and the electron scanning micrographs were made by a specialised laboratory in the university.

\section{RESULTS AND DISCUSSION}

\subsection{General screening and comparison of high and low methoxylated pectins}

\subsubsection{Molecular and material properties}

First impressions of differences between both pectin groups give the electron scanning micrographs in Figure 1. The HMP particles had a visibly rougher surface and were more porous than the according LMP. Methoxylation in industrial scale is often made in an acidic environment ${ }^{1}$ where the majority of free carboxylic groups are undissociated. The pectin macromolecules show low electrostatic repulsion and are able to form many strong interand intramolecular hydrogen bonds. The result is a compact structure that is trapped during drying in a partly crystalline state and can negatively influence the dissolution properties. The main problem in the hydration and dispersion of pectin powder is to limit the "fish-eye effect". Some of the tested pectins were really difficult to dissolve (Table 1) and confirmed this experience. It took mostly more than 20 min and sometimes even more than $1 \mathrm{~h}$ to dissolve $100 \mathrm{mg}$ pectin in $50 \mathrm{ml}$ distilled water, and the smoother surface and a partly crystalline state of LMP particles (in comparison to HMP) additionally delayed the necessary hydration process.

Another general result of the demethoxylation procedure was the smaller chain length of the pectins. The intrinsic viscosity (IV) of the tested LMP was significantly lower than that of the according HMP (Table 1). The reason is that any chemical demethoxylation, independent on acidic or alkaline conditions, does not only cleave the ester bonds but also, to a certain extent, the glycosidic linkages in the galacturonic acid backbone.

The third general difference between HMP and LMP was the colour, in particular the bvalue (Table 1). LMP of company 1 and 2 hade a significantly higher $+b$-value (yellow) than the according HMP, in case of company 3 this effect, however, was not clear. It seems that more unsaturated uronides developed during demethoxylation in company 1 and 2 which were able to form brown-coloured reaction products whereas in company 3 this was partly prevented. These differences between the companies were confirmed by the galacturonan content $(\mathrm{GC}=$ purity). Intensive chemical treatment can cause not only browning unsaturated uronides but also removes neutral polysaccharides and impurities and, thus, 
increases the GC. The difference in GC between HMP and LMP of company 3 were smaller than in case of company 1 or 2 (Table 1).

The differences in particle size and size distribution between HMP and LMP (Table 1) depended neither clearly on the DM nor on the pectin company, but probably mainly on the milling equipment and conditions. The particle size should be, however, not completely neglected by the pectin producers because of its influence on dissolution and application as discussed above.
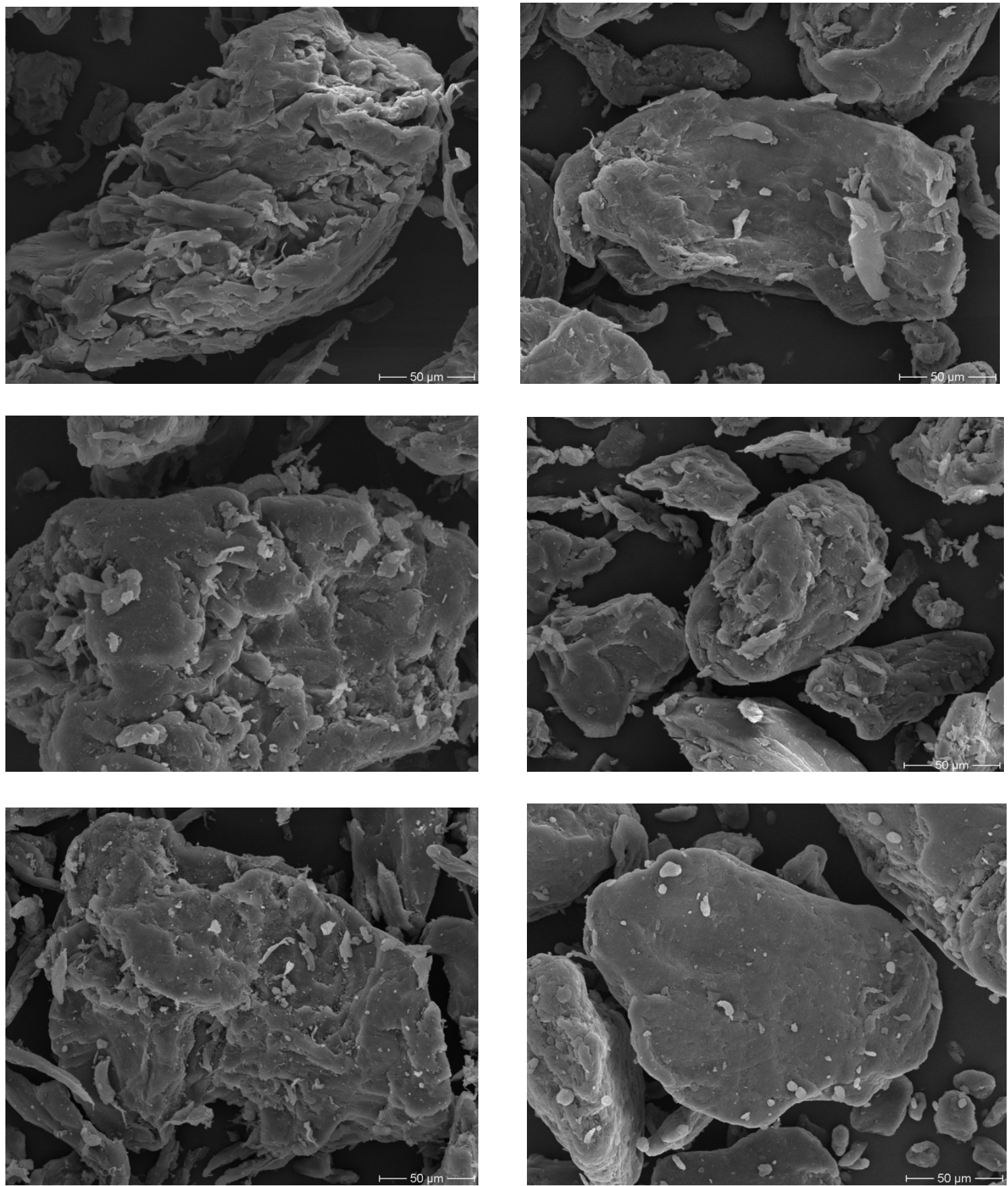

Figure 1 Electron scanning microscopy of HMP (left side) and LMP (right side) of the three different companies. First line $=$ company 1 , second line $=$ company 2 , third line $=$ company 3. 
Table 1: Molecular parameters, material and gelling properties of the tested pectins

$G C=$ galacturonan content, $D M$ degree of methoxylation, $I V=$ intrinsic viscosity, $E_{500}=$ absorption at $500 \mathrm{~nm}$, $A_{o}=$ particle surface, $T_{p D S C}=$ peak temperature in DSC signal, $T_{\text {on }}$ and $T_{p D T G}=$ extrapolated onset- and peak temperature in DTG signal, $\Delta T=$ peak width, $v_{\text {max }}=$ maximum degradation velocity, GP=gelling point, IST=initial structuring temperature, CST=critical structuring temperature, $G^{\prime}$ end $=$ final storage modulus, tan $\delta_{\text {end }}=$ final loss factor.

\begin{tabular}{|c|c|c|c|c|c|c|c|c|c|c|c|c|c|c|c|c|c|c|c|c|c|c|}
\hline \multirow[b]{2}{*}{ source } & \multirow[b]{2}{*}{ sample } & \multicolumn{3}{|c|}{$\begin{array}{c}\text { molecular } \\
\text { parameters }\end{array}$} & \multicolumn{3}{|c|}{ colour } & \multicolumn{3}{|c|}{ dissolution } & \multicolumn{2}{|c|}{$\begin{array}{l}\text { particle } \\
\text { analysis }\end{array}$} & \multicolumn{5}{|c|}{ thermal degradation } & \multicolumn{5}{|c|}{ gelation } \\
\hline & & $\begin{array}{c}\text { GC } \\
\%\end{array}$ & $\begin{array}{c}\text { DM } \\
\%\end{array}$ & $\begin{array}{c}\text { IV } \\
\mathrm{cm}^{3} / \mathrm{g}\end{array}$ & $\mathbf{L}$ & $\mathbf{a}$ & b & $\begin{array}{l}\text { time } \\
\text { min }\end{array}$ & $\mathbf{E}_{500}$ & pH & $\begin{array}{c}\text { median } \\
\mu \mathrm{m}\end{array}$ & Ao & $\begin{array}{c}\mathbf{T}_{\mathrm{pDSC}} \\
{ }^{\circ} \mathbf{C}\end{array}$ & $\begin{array}{c}\text { TonDTG }_{\text {on }} \\
{ }^{\circ} \mathbf{C}\end{array}$ & $\begin{array}{c}\mathbf{T}_{\mathbf{p D T G}} \\
{ }^{\circ} \mathbf{C}\end{array}$ & $\begin{array}{r}\Delta \mathrm{T} \\
\mathbf{K}\end{array}$ & $\begin{array}{c}V \max \\
\% / \min \end{array}$ & $\begin{array}{l}\text { GP } \\
{ }^{\circ} \mathbf{C}\end{array}$ & $\begin{array}{l}\text { IST } \\
{ }^{\circ} \mathrm{C}\end{array}$ & $\begin{array}{c}\mathrm{CST} \\
{ }^{\circ} \mathrm{C}\end{array}$ & $\begin{array}{c}\mathbf{G}_{\text {end }}^{\prime} \\
\mathbf{P a}\end{array}$ & \\
\hline \multirow[t]{3}{*}{1} & $\mathbf{1 A}$ & 89.3 & 60.9 & 639 & 86 & & 12.9 & 15 & 0048 & 4.04 & 145 & 607 & 2405 & 2204 & 2278 & 27.6 & 19.9 & & & & & \\
\hline & 1B & 85.5 & 59.6 & 598 & 89.7 & 1.1 & 10.9 & $>60$ & 0.045 & 3.62 & 178 & 437 & 239.4 & 220.6 & 236.1 & 25.8 & 22.2 & 90.5 & 93.2 & 81.0 & 1077 & 0.078 \\
\hline & $1 \mathrm{C}$ & 93.7 & 24.2 & 318 & 90.0 & 1.5 & 16.9 & 30 & 0.022 & 3.52 & 110 & 905 & 238.4 & 217.3 & 233.8 & 28.5 & 17.3 & 51.2 & 53.0 & 42.0 & 91 & 0.151 \\
\hline \multirow[t]{3}{*}{2} & $\mathbf{2 A}$ & 81.6 & 68.9 & 647 & 6.4 & 2.0 & 12.2 & 20 & 0.131 & 3.42 & 101 & 1118 & 244.0 & 220.5 & 240.3 & 35.6 & 16.3 & 86.0 & 88.0 & 84.5 & 815 & 0.068 \\
\hline & $2 B$ & 87.7 & 55.1 & 492 & 87.7 & 1.6 & 11.4 & 30 & 0.166 & 3.45 & 96 & 1154 & 245.0 & 220.4 & 240.7 & 34.5 & 16.2 & 57.6 & 61.5 & 57.5 & 639 & 0.054 \\
\hline & $2 C$ & 91.5 & 30.1 & 358 & 81.2 & 3.4 & 24.0 & 30 & 0.442 & 3.18 & 82 & 1244 & 246.7 & 221.0 & 241.0 & 33.5 & 15.8 & 42.9 & 51.0 & 36.5 & 81 & 0.200 \\
\hline \multirow[t]{8}{*}{3} & $\mathbf{3 A}$ & 80.9 & 69.8 & 554 & 89.9 & 1.4 & 11.0 & 25 & 0.056 & 3.48 & 101 & 1114 & 249.6 & 231.7 & 246.1 & 24.3 & 22.2 & 76.7 & 79.0 & 76.0 & 587 & 0.061 \\
\hline & 3B & 83.4 & 57.1 & 576 & 88.4 & 1.7 & 12.9 & $>60$ & 0.054 & 3.56 & 81 & 1570 & 247.9 & 228.7 & 243.9 & 25.7 & 20.7 & 56.5 & 59.5 & 56.0 & 877 & 0.051 \\
\hline & $3 C$ & 81.5 & 63.6 & 608 & 88.5 & 1.8 & 14.0 & $>60$ & 0.028 & 3.55 & 98 & 1113 & 249.6 & 231.1 & 245.8 & 24.7 & 21.4 & 67.1 & 70.0 & 65.5 & 788 & 0.057 \\
\hline & 3D & 84.8 & 32.8 & 363 & 87.7 & 1.1 & 13.0 & $>60$ & 0.022 & 5.24 & 118 & 1127 & 230.9 & 213.3 & 228.4 & 54.0 & 17.2 & 43.4 & 59.5 & 30.5 & 44 & 0.166 \\
\hline & $3 E$ & 81.5 & 30.2 & 336 & 84.0 & 2.7 & 20.1 & $>60$ & 0.039 & 4.91 & 149 & 523 & 234.1 & 217.0 & 231.6 & 50.4 & 17.8 & 57.6 & 66.0 & 55.0 & 128 & 0.128 \\
\hline & $3 F$ & 78.5 & 27.7 & 327 & 86.9 & 1.5 & 15.0 & 45 & 0.040 & 5.24 & 103 & 1295 & 233.9 & 216.1 & 231.4 & 46.9 & 15.6 & & 78.5 & 69.0 & 293 & 0.120 \\
\hline & $3 G$ & 82.3 & 69.0 & 518 & 84.0 & 2.8 & 15.6 & 45 & 0.040 & 3.64 & 112 & 956 & 248.2 & 229.6 & 244.4 & 25.1 & 22.0 & 69.8 & 72.0 & 69.0 & 296 & 0.090 \\
\hline & $3 H$ & 87.0 & 56.5 & 529 & 88.2 & 1.8 & 12.9 & $>60$ & 0.065 & 3.66 & 89 & 1129 & 246.2 & 226.0 & 242.3 & 27.0 & 20.3 & 54.0 & 54.0 & 53.0 & 348 & 0.087 \\
\hline
\end{tabular}




\subsubsection{Thermal analysis}

A further method for a quick evaluation of pectin is the thermal analysis as combination of differential scanning calorimetry DSC and thermogravimetry TG. The DSC signals give information on transition and degradation enthalpies. The TG and its first derivation DTG allow insight into thermal stability by the extrapolated temperatures $\mathrm{T}_{\text {on DTG }}$ and into homogeneity by the degradation time, measured as peak width $\Delta \mathrm{T}$, both from the DTG signal. The values are given in Table 1 and some typical signals in Figure 2.
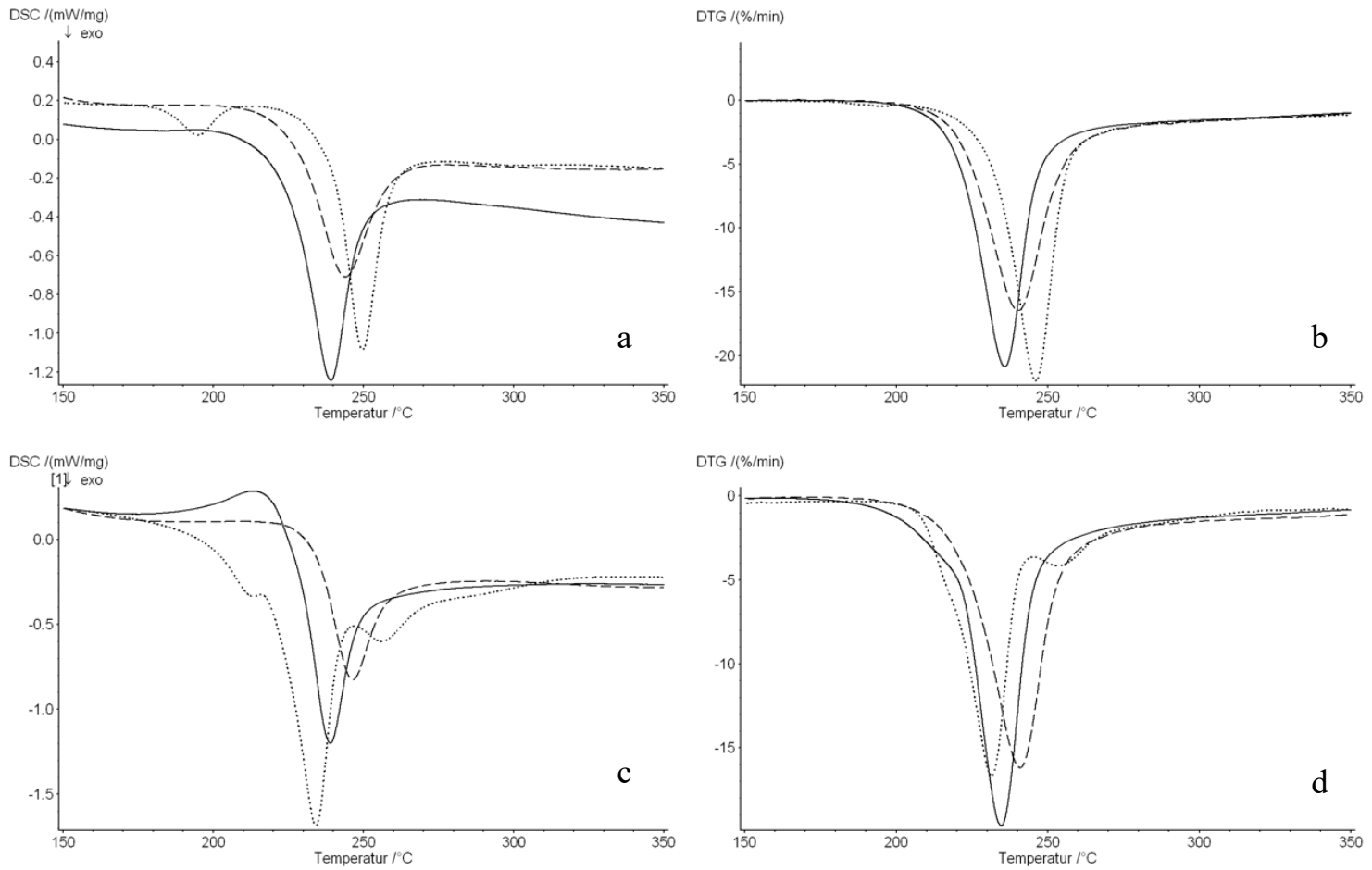

Figure 2 DSC signal (a,c) and DTG signals $(b, d)$ of HMP $(a, b)$ and LMP $(c, d)$. $-=$ company $1,---=$ company $2, \cdots \bullet=$ company 3 .

Comparing HMP and LMP, the main differences are clearly visible: The two HMP of company 1 and 3 were more similar (DTG peak form) and homogenous (peak width) than the according LMP. In case of company 2 these differences were smaller.

Comparing the DSC signals of the three HMP in detail, the pectins of company 1 and 2 were similar and had only one exothermic degradation peak. The HMP of company 3 , however, had another smaller exothermic peak before the main fraction degradation and also a small weight loss in the according DTG signal; that means another component beside the normal pectin in this sample was degraded. The HMP of company 2 seemed to be the least homogenous; the peak width in DTG was about $10 \mathrm{~K}$ higher than that of the two others. Nevertheless, the DTG signals of the 3 HMP were partly similar.

Comparing the three LMP, the differences were even clearer. LMP of company 1 had an endothermic pre-peak with only small weight loss; it might result mainly from a conformation transition as discussed elsewhere. ${ }^{5}$ The LMP of company 3 had the most shoulders and peaks in DSC as well as DTG signals and the broadest peaks, it was the least homogenous.

The DSC and DTG signals of the three companies were representative (= found for several single samples). They were a kind of a "fingerprint" and allowed an easy detection of potential differences in the pectin production process which were discussed in the analysis 
of molecular parameters. Unfortunately, the processing details of the single materials were more or less secret and it was not possible to confirm the assumptions about differences in technology. Nevertheless, it is generally possible to use the thermal analysis for a quick screening in quality control for detection of changes in quality and processing.

\subsubsection{Gelling properties}

The structuring process parameters and the gel properties of the tested pectins were not completely comparable because of different gel compositions and gelation mechanisms. Nevertheless, structuring temperatures of HMP were higher than those of LMP with one exception in company 3 . The final values of $G^{\prime}$ (solid-like properties) were higher for almost all HMP than for the according LMP and those of $\tan \boldsymbol{\delta}$ (brittleness) were definitely higher for the LMP.

\subsection{Comparison of single pectins with similar degree of methoxylation}

The degree of methoxylation is often used as a key parameter for pectin application. The following examples show, however, that pectins with comparable molecular parameters (especially nearly identical DM) of different companies and even from one company but different production periods were far from similar in their material properties and gelling behaviour. The examples were the HMP 2A, 3A and 3G with DM about $69 \%$ and the LMP $2 \mathrm{C}$ and $3 \mathrm{E}$ with DM $30 \%$, for the detailed values see Table 1.

Beside the general effects of demethoxylation as discussed above, there were several specific differences. The first can be seen from the thermal analysis as discussed in 3.1.2 above. In particular, the HMP of company 2 was less thermal stable and less homogenous than the according samples from company 3 . In case of the LMP, the tendencies were just opposite in thermal stability but the same with respect to homogeneity. These were the first indicators of different processing parameters and resulting properties.

The intrinsic viscosities were higher for the pectins from company 2, what allowed the conclusion that this company prepared their pectins under conditions (especially $\mathrm{pH}$ and $\mathrm{T}$ ), which caused less cleaving of bonds in the backbone. Another difference was the high galacturonan content (purity) of the LMP 2C, most of neutral sugars and impurities were removed during demethoxylation. Such an effect was found also for company 1 and it is known from laboratory scale pectin modifications, too, where it was found especially after acid treatments.

The most interesting differences with high practical relevance were found for the structuring process and the gel properties. In case of HMP, the structuring temperatures varied not only between the two companies for about $10 \mathrm{~K}$ but also for about $7 \mathrm{~K}$ between $3 \mathrm{~A}$ and $3 \mathrm{G}$ from one company but different years (Figure 3 ). Also the end level of the storage modulus $\mathrm{G}^{\prime}$ end and of the loss factor tan $\delta_{\text {end }}$ differed not only between the two companies but also within one company. In case of the LMP, the gelpoint could not be determined for all samples, the structuring temperatures were, however, clearly higher for company 3 . The LMP-gel of company 3 was more solid (higher $\mathrm{G}^{\prime}$ ) and brittle (lower $\tan \delta$ ) than that of company 2. 


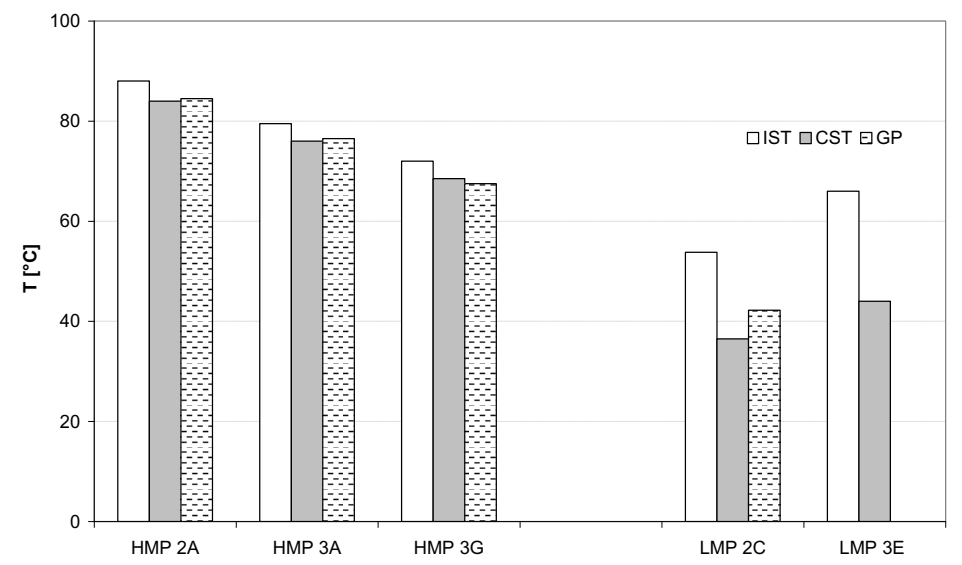

Figure 3 Structuring temperatures of HMP and LMP.

$I S T=$ initial structuring temperature, $C S T=$ critical structuring temperature, $G P=$ gelpoint .

\subsection{Statistical analysis}

The statistical tests for interdependencies of molecular parameters, material properties and structuring were made by comparing either all pectins together, or the groups of HMP and LMP as well as 8 pectins from one company separately (Tab.2). As can be clearly seen, DM was the key parameter for many other properties. It had not only the significant well-known impact on the gelpoint but also on intrinsic viscosity (molecular weight) as discussed above. The interaction of DM and thermal stability was found only for the pectins of one company, this confirmed the results of a previous examination of model pectins. ${ }^{4}$ Influences of pectin purity (GC) and molecular weight (IV) were found mainly in the LMP-group and are probably indirectly influenced by the DM.

Table 2 Statistical analysis of the interactions of molecular parameters and material and structuring properties.

$X X X=R>0.9, X X=R>0.8$. For the single parameters see Table 1.

\begin{tabular}{|c|c|c|c|c|}
\hline & $\begin{array}{c}\text { all } \\
\text { pectins } \\
\mathrm{n}=32\end{array}$ & $\begin{array}{c}\text { all HMP } \\
n=20\end{array}$ & $\begin{array}{c}\text { all LMP } \\
n=12\end{array}$ & $\begin{array}{c}\text { one company } \\
n=18\end{array}$ \\
\hline$\overline{D M}-\mathrm{IV}$ & \multirow{5}{*}{$\begin{array}{c}\mathrm{xxx} \\
\mathrm{xx}\end{array}$} & \multirow{5}{*}{$\begin{array}{c}x x x \\
x x \\
x x x\end{array}$} & \multirow{5}{*}{$\mathrm{XXX}$} & $\mathrm{xxx}$ \\
\hline DM - GP & & & & $\mathrm{xxx}$ \\
\hline DM - IST & & & & \\
\hline DM - CST & & & & \\
\hline $\mathrm{DM}-\mathrm{Tp}_{\mathrm{DTG}}$ & & & & $\mathrm{xxx}$ \\
\hline GC - GP & \multirow[t]{3}{*}{$\mathrm{xx}$} & & & \\
\hline GC - IST & & & $\mathrm{xx}$ & \\
\hline $\mathrm{GC}-\mathrm{Tp} \mathrm{p}_{\mathrm{DTG}}$ & & & $\mathrm{xx}$ & \\
\hline IV - GP & \multirow[t]{4}{*}{$\mathrm{xx}$} & & \multirow{4}{*}{$\begin{array}{l}\mathrm{xxx} \\
\mathrm{xxx}\end{array}$} & \multirow[b]{4}{*}{$\mathrm{XXX}$} \\
\hline IV - IST & & & & \\
\hline IV - CST & & & & \\
\hline IV - Tp $p_{\text {DTG }}$ & & & & \\
\hline GP - IST & $\mathrm{xx}$ & $\mathrm{xxx}$ & & \multirow[b]{2}{*}{ XXX } \\
\hline GP - CST & XXx & $\mathrm{xxx}$ & & \\
\hline
\end{tabular}


The correlations of the classical gelpoint and the new structuring parameters IST and CST are of special interest. It should be considered, however, that a gelpoint could be determined not for all LMP. The correlation of gelpoint and CST was significant for all categories except LMP; that supports the application of CST as complementation to or instead of the gelpoint and is highly important, in particular for gelation processes without clear gelpoints like sometimes were found in LMP.

\section{CONCLUSIONS}

The presented results confirmed the general influence of processing parameters on the pectin quality and application for a collection of commercial pectins from different companies, that were found before for model pectins made from one original sample. These parameters such as $\mathrm{pH}$, demethoxylation temperature and drying conditions - influenced the molecular weight, colour, purity, thermal stability and homogeneity of the materials. Moreover, they determined the state (amorphous or crystalline) and material properties (surface quality and porosity) of the pectin powder particles, which are crucial for the application properties such as dissolution and gelation.

The degree of methoxylation is a key factor for many tested pectin properties, especially in the gelation process. But it is not the only factor what was revealed by a comparison of pectins with similar DM.

The thermal analysis proved to be a helpful and rapid screening method for pectin characterisation. Differences, found in DSC and TG, were confirmed by analysis of molecular parameters.

Any pectin producing company should try to promote the favourable material properties, such as rough porous particles and an amorphous state with easily cleavable inter- and intramolecular interactions, in order to produce pectins with excellent application properties.

\section{References}

1 C. Rolin, Commercial pectin preparations in Pectins and their Manipulation, eds. G.B. Seymour, J.P. Knox, Blackwell Publishing, Oxford, 2002, pp. 222-241.

2 A.G.J. Voragen, W. Pilnik, J.-F. Thibault, M.A.V. Axelos, C.M.G.C. Renard, Pectins in Food Polysaccharides and their applications, ed. A.M Stephen, M. Dekker, New York, 1995, chapter 10, pp. 287-339.

3 B.R. Thakur, R.K. Singh, A.K. Handa, Critical Rev. Food Sci. Nutrition, 1997, 37, 47.

4 U. Einhorn-Stoll, H. Kunzek, G. Dongowski, Food Hydrocolloids, 2007, 21, 1101.

5 U. Einhorn-Stoll, H. Kunzek, Food Hydrocolloids, 2009, 23, 40.

6 U. Einhorn-Stoll, T. Salazar, B. Jaafar, H. Kunzek, Nahrung/Food, 2001, 45, 332.

7 H. Kastner, U. Einhorn-Stoll, B. Senge, Gums and stabilisers for the food industry, 2011, 16. 